\section{P2.147 COMMUNITY ENGAGEMENT, THE BEST ANTIRETROVIRAL}

doi:10.1136/sextrans-2013-051184.0411

C M Ngelese, B B Bakashaba. The AIDS support organization uganda, kampala, Uganda

Background As antiretroviral treatment continues to expand, ensuring patient retention over time is an increasingly important concern. This, together with capacity and human resource constraints, has led to the consideration of community drug distribution point (CDDP) model for the delivery of antiretroviral therapy (ART). In 2008, TASO Rukungiri launched a community model of ART distribution and adherence monitoring by community groups in Rukungiri district.

Description Patients who are stable on ART for 3 months are informed about the community ART group model and invited to join the groups. Group members have 4 key functions: facilitate monthly ART distribution to other group members in the community, provide adherence and social support, monitor outcomes, and ensure each group member undergoes a clinical consultation at least once every 6 months. Group members visit the centre on a rotational basis, such that each group member has contact with the service centre every 6 months. Drugs are given on two monthly bases. At every six month visit at the service centre every client is done the following tests: $\mathrm{CD}_{4}$ count, $\mathrm{h}$ b levels, viral load, Lfts and Rfts.

Results Between February 2009 and May 2011, 3000 members were enrolled into 69 groups. Median follow-up time within a group was 12.9 months (IOR 8.5-14.1). During this time, 180 (6\%) were transferred out, 2820 patients still in community groups, 2749 $(97.5 \%)$ were remaining in care, $56(2 \%)$ had died, and $6(0.2 \%)$ were lost to follow-up.

Lessons learnt: Decongestion at the service centre, easy access, monitoring, adherence and quality service, finally proper documentation.

Conclusion The Community ART Group model was initiated by TASO to improve access, patient retention, and decongest centre services. Early outcomes are highly satisfactory in terms of mortality and retention in care, lending support to such out-of-clinic approaches.

\section{P2.148 UPTAKE AND EVALUATION OF AN ELECTRONIC LEARNING TOOL FOR SEXUALLY TRANSMITTED INFECTIONS AND HIV}

doi:10.1136/sextrans-2013-051184.0412

\section{J Sherrard. Oxford University Hospitals NHS Trust, Oxford, UK}

Background An elearning tool: eHIV-STI was developed by the British Association for Sexual Health and HIV (BASHH), the Royal Colleges of Physicians (RCP) and e-Learning for Healthcare. The first sessions went live in February 2010 and the project was completed in December 2011 with 141 sessions. eHIV-STI covers the knowledge component of the UK Genitourinary Medicine (GUM) training curriculum, from introductory to advanced STI and HIV knowledge required for practise as a specialist. It also acts a refresher for established consultants and has been approved by the RCP for external CPD, supporting revalidation. It is available free to all clinical staff in the NHS

Introductory sessions have been embedded in STI training for non specialists, and for GUM trainees, since 2012, sessions are used to complement national specialist courses, delivering some knowledge in advance by allowing the face to face sessions to deal with more complex issues.

Methods Using reports generated within the learning management system we undertook detailed analysis of usage of the tool since its introduction, by clinician type, sessions accessed, and evaluation of sessions, as well as collating more specific feedback from trainees using it as an adjunct to training courses.
Results The number of registered users has risen steadily since 2010 to 10217 individual users accessing sessions 104857 times by the end of 2012. The majority of GUM trainees and consultants are registered users, but additionally it is used extensively across the health service including 2341 nurses, 1122 newly qualified doctors and 1259 general practitioners.

Evaluation of the material ranges from 4.3 to 4.6 out of 5 for content, presentation, interactivity, and self assessment.

Conclusion eHIV-STI has rapidly become a very well used and evaluated learning tool for clinicians working in the fields of sexual health and HIV and in allied areas.

\section{P2.149 ELECTRONIC MEDICAL RECORDS ARE PREFERRED BY CLINICIANS AND ASSOCIATED WITH IMPROVED PATIENT FLOW AT A LARGE URBAN SEXUAL HEALTH CENTRE}

doi:10.1136/sextrans-2013-051184.0413

'L A Vodstrcil, 1,2C K Fairley, 1,2S Huffam, ${ }^{2} \mathrm{R}$ Cummings, ${ }^{1,2} \mathrm{M}$ Y Chen, ${ }^{2} \mathrm{G}$ Fehler, ${ }^{1,2} \mathrm{C} \mathrm{S}$ Bradshaw, ${ }^{2} \mathrm{~T}$ Schmidt, ${ }^{2} \mathrm{~K}$ Berzins, ${ }^{1} \mathrm{~J}$ S Hocking. ${ }^{~}$ Melbourne School of Population and Global Health, University of Melbourne, Parkville, Australia; ${ }^{2}$ Melbourne Sexual Health Centre, Carlton, Australia

Background Electronic Medical Record (EMR) functions include electronic ordering of investigations and receiving results, electronic prescribing, recording of clinical information and decision support software. Despite substantial investment in EMR systems, there has been little research to evaluate them. Our aim was to evaluate changes in efficiency and quality of services after the introduction of a purpose built EMR system, and to assess its acceptability by the doctors, nurses and patients using it.

Methods We compared the number of patients seen per hour within nine month periods before and after the introduction of an EMR system in a large sexual health service, in Melbourne Australia. A sample of records from both EMR and Paper Medical Record (PMR) periods were audited by two physicians to evaluate quality and completeness of records. Staff and patients completed anonymous surveys about their satisfaction and experience with the EMR system.

Results There were 9,752 doctor consultations (in 5,512 consulting hours) in the PMR period and 9,145 doctor consultations (in 5,176 consulting hours) in the EMR period eligible for inclusion in the analysis. There were $5 \%$ more consultations per hour seen by doctors in the EMR period compared to the PMR period (rate ratio $=1.05 ; 95 \%$ confidence interval $(\mathrm{CI}) ; 1.02-1.08)$ after adjusting for type of consultation. The qualitative evaluation of 300 records for each period showed no difference in quality or completeness $(\mathrm{P}$ $>0.17)$. The survey of clinicians demonstrated that doctors and nurses preferred the EMR system $(\mathrm{P}<0.01)$ and the patient survey in each period showed no difference in satisfaction of their care (97\% for PMR, 95\% for EMR, $\mathrm{P}=0.61$ ).

Conclusion The introduction of an integrated EMR improved efficiency while maintaining the quality of the patient record. The EMR was popular with staff and was not associated with a decline in patient satisfaction in clinical care provided.

\section{P2.150 PATTERNS OF STI CLINIC UTILIZATION AMONG NON- MIGRANT AND MIGRANT FEMALE SEX WORKERS IN KARNATAKA, INDIA}

doi:10.1136/sextrans-2013-051184.0414

'M L Becker, ${ }^{2}$ S Mishra, ${ }^{3} S$ Ramanaik, ${ }^{4} S$ Halli, ${ }^{1} \mathrm{~J}$ Blanchard, Payana Study Group. ${ }^{1}$ Centre for Global Public Health, University of Manitoba, Winnipeg, MB, Canada, ${ }^{2}$ Department of Infectious Disease Epidemiology, Imperial College, London, UK ${ }^{3}$ Karnataka Health Promotion Trust, Bangalore, India; ${ }^{4}$ University of Manitoba, Winnipeg, $M B$, Canada 